\title{
ORIGINAL RESEARCH \\ Pituitary Iron and Volume Imaging in Healthy Controls
}

L.J. Noetzli

A. Panigrahy

A. Hyderi

A. Dongelyan

T.D. Coates

J.C. Wood
BACKGROUND AND PURPOSE: Patients with transfusional iron overload develop iron deposits in the pituitary gland, which are associated with volume loss and $\mathrm{HH}$. The purpose of this study was to characterize R2 and volumetric data in a healthy population for diagnostic use in patients with transfusional iron overload.

\begin{abstract}
MATERIALS AND METHODS: One hundred healthy controls without iron overload between the ages of 2 and 48 were recruited to have MR imaging of the brain to assess their pituitary R2 and volume. Pituitary R2 was assessed with a 8-echo spin-echo sequence, and pituitary volumes, by a 3D spoiled gradient-echo sequence with 1- $\mathrm{mm}^{3}$ resolution. A 2-component continuous piecewise linear approximation was used for creating volumetric and R2 nomograms. Equations were generated from regression relationships for convenient $z$-score calculation.
\end{abstract}

RESULTS: Pituitary R2 rose weakly with age $\left(r^{2}=0.19, P<.0001\right)$. Anterior and total pituitary volumes increased steadily up to 18 years of age, after which volume slightly decreased. Females had larger pituitary glands, most likely representing their larger lactotroph population.

CoNCLUSIONS: From these data, a clinician can calculate the $z$ scores for R2 and pituitary volume in patients with iron overload. Normal ranges are well-differentiated from values previously associated with endocrine disease in transfusional siderosis; this finding suggests that preclinical iron overload can be recognized and appropriately treated.

ABBREVIATIONS: GnRH = gonadotropin-releasing hormone; HH = hypogonadotropic hypogonadism; $R 2$ = pituitary iron $l^{2}$ ron overload is a major concern for chronically transfused patients with hemoglobinopathies such as thalassemia major. ${ }^{1}$ Despite advances in chelation therapy, chronic transfusions result in iron deposition in many organs. ${ }^{2}$ The liver is the main storage depot for iron and is a surrogate for total body iron. ${ }^{3}$ However, extrahepatic organs such as the heart, pancreas, and pituitary gland are the most vulnerable to the harmful effects of iron. ${ }^{2,4}$ Iron overload in extrahepatic organs accounts for morbidities such as cardiomyopathy, diabetes, and $\mathrm{HH}$; secondary amenorrhea and $\mathrm{HH}$ are the most common endocrinopathies in thalassemia major. ${ }^{2,4,5}$

One of the reasons that $\mathrm{HH}$ is so prevalent is that the effects of gonadotrope iron deposition are clinically silent until puberty. While GnRH stimulation testing can be used in postpubertal patients to assess pituitary gland function, immature glands do not normally respond to $\mathrm{GnRH}$ provocation, even

Received March 10, 2011; accepted after revision June 14

From the Department of Pediatrics (L.J.N., A.H., J.C.W.), Division of Cardiology, and Department of Pediatrics (A.D., T.D.C.), Division of Hematology-Oncology, Children's Hospital Los Angeles, Los Angeles, California; and Department of Radiology (A.P.), Children's Hospital of Pittsburgh, Pittsburgh, Pennsylvania.

This work was supported by research grants from Novartis Pharmaceuticals, Cooley's Anemia Foundation, the General Clinical Research Center at Children's Hospital Los Angeles Satellite of the University of Southern California (National Institutes of Health RR00043-43), and the Centers for Disease Control (Thalassemia Center Grant U27/CCU922106).

Paper previously presented in part as a poster at: American Society of Hematology, December 5-8, 2009; New Orleans, Louisiana.

Please address correspondence to John Wood, MD, PhD, Department of Cardiology, Children's Hospital Los Angeles, 4650 Sunset Blvd., Mail Stop \#34, Los Angeles, CA 90027; e-mail: jwood@chla.usc.edu

Indicates open access to non-subscribers at www.ajnr.org

http://dx.doi.org/10.3174/ajnr.A2788 in healthy patients. Thus, surrogates for preclinical R2 deposition and damage are imperative. MR imaging can be used to measure $\mathrm{R} 2^{6,7}$ and volume, analogous to its use for the heart, liver, and pancreas. ${ }^{8,9}$

Several studies have explored the need for pituitary MR imaging monitoring in patients with iron overload. The presence of $\mathrm{R} 2$ in thalassemia major has been confirmed by studies that show decreased pituitary $\mathrm{T} 2$ relaxation rates compared with controls. ${ }^{7,10}$ Furthermore, increased R2 and decreased pituitary height have been shown to predict clinical and biochemical HH in adults with thalassemia major. ${ }^{6,7,11}$ There has also been evidence that increased R2 precedes pituitary volume loss and hypogonadism. ${ }^{12}$ We have demonstrated that R2 deposition can be seen in children as young as 4 years of age, with volume loss beginning in the second decade of life. ${ }^{13}$ These findings suggest that proper MR imaging screening may identify iron-mediated $\mathrm{HH}$ before it is clinically observable in at-risk patients. At our institution, patients with transfusional iron overload have annual MR imaging to assess their hepatic and cardiac iron burdens from the age of 2 onward. Thus, MR imaging is a logical way to also assess R2 overload and gland volume.

To interpret R2 and volumetric data in the iron-overloaded population, it is necessary to have age- and sex-appropriate norms. Normative data for R2 are sparse, with little pediatric data. Normal pituitary volume has been measured to identify pituitary changes related to bipolar disorder or traumatic brain injury, including 3 large pediatric studies. ${ }^{14-16}$ However, no study to date is suitable for serial $z$-score calculation through the transition from childhood to young adulthood, when $\mathrm{HH}$ becomes clinically evident. ${ }^{17-19}$ The purpose of this study was to establish nomogram equations for R2 and gland volume that would allow continuous $z$-score estimates 
for children and adults with transfusional siderosis. Pituitary R2 was used as the metric for R2 because it is robust to susceptibility artifacts and more reproducible across platforms than signal-intensity ratio or $\mathrm{R} 2 *$ techniques.

\section{Materials and Methods}

One hundred subjects without iron overload (51 females and 49 males) between the ages of 2 and 48 were recruited for MR imaging of the brain to assess their R2 and volume. Subjects as young as 4 years of age were studied without anesthesia by using video goggles and appropriate coaching. Seven subjects who were younger than of 4 years of age and could not undergo an unsedated MR imaging were studied under general anesthesia by adding the research MR imaging ( $<10$ minute duration) onto a clinically indicated sedated head MR imaging; the clinical indication for head MR imaging was seizure disorder or developmental delay. No structural abnormalities were identified in these 7 studies. All other subjects were recruited from the general public by approved flyers and verbal communication with hospital staff, their friends, and family. Participants were questioned regarding prior or current illnesses, hospitalizations, and medications. Subjects were excluded for any chronic illnesses or pathologies known to affect pituitary gland size or structure. This study was approved by the Committee of Clinical Investigation at Children's Hospital Los Angeles (CCI 08-00143). Informed consent was obtained for all subjects.

Imaging was performed by using an 8 -element head coil on a $1.5 \mathrm{~T}$ Achieva system running software release 2.5 (Philips Healthcare, Best, the Netherlands). Following standard localizers, a coronal 3D fastspoiled gradient-echo image was obtained by using 1-mm isotropic voxels, a slab thickness of $12-16 \mathrm{~cm}$, and an FOV of 20-24 cm; images were 2 -fold Fourier-interpolated in-plane for reconstructed voxel sizes of $0.5 \times 0.5 \times 1 \mathrm{~mm}$. TR was $7 \mathrm{~ms}$, TE was $3.2 \mathrm{~ms}$, flip angle was $8^{\circ}$, and bandwidth was $241 \mathrm{~Hz} /$ pixel. Pituitary R2 was measured by using multiple-echo spin-echo images collected in a single midline sagittal plane and a single coronal plane centered on the anterior pituitary gland. Imaging parameters were a TR of $500 \mathrm{~ms}$; flip angles $90^{\circ}-180^{\circ}-180^{\circ} \ldots$; TEs of $15,30,45,60,75,90,105$, and $120 \mathrm{~ms}$; 1-mm in-plane isotropic resolution; and 3-mm section thickness.

Anterior and posterior pituitary volumes were manually planimetered directly on the Synapse imaging PACS (Fuji Medical Systems, Stamford, Connecticut); cross-sectional areas were converted to volumes by multiplying by the section thickness and summed across the gland. All anatomic boundaries were confirmed by a single boardcertified neuroradiologist. Because this technique of acquiring and calculating pituitary volume is time-consuming, we investigated simpler metrics to estimate gland size. We measured the area and length of the pituitary gland from the first echo from the coronal and sagittal R2 images. Anterior and total pituitary volumes were calculated by using a 5/6 area-length measurement as follows:

1) Volume $=(5 / 6) *$ Area $_{\text {sag }} *$ Area $_{\text {cor }} /$

$$
\text { [1/2] }\left(\text { Height }_{\text {sag }}+\text { Height }_{\text {cor }}\right) \text {. }
$$

Gland height was calculated at the intersection of the imaging planes. We also explored a biplane estimate of anterior pituitary height, calculated as follows:

2) Height $=[1 / 2]\left(\right.$ Area $_{\text {sag }} /$ Length $_{\mathrm{AP}}+$ Area $_{\text {cor }} /$ Length $\left._{\mathrm{RL}}\right)$.

We used an equivalent height (Area/Length) rather than a simple planimetered height to reduce the variability introduced by different gland orientations; this procedure markedly stabilized the estimates. Biplane measurements exhibited significantly lower variance with age than either single-plane measurement.

Pituitary R2 was calculated on a pixelwise basis from the coronal and sagittal multiecho spin-echo images. Each pixel was fit to a simple monoexponential across all echoes, creating a R2 map; no offset correction was needed. The entire visible anterior pituitary gland was traced in the R2 map, yielding a distribution of R2 values, from which mean and median values were retained for statistical considerations. Mean and median values were compared with one another by using Bland-Altman analysis. Bland-Altman statistics were also used to compare coronal and sagittal acquisitions.

Many strategies were considered for creating volumetric and R2 nomograms; however, a 2-component continuous piecewise linear approximation was used for the following reasons: 1) Pituitary volume increased linearly with age from birth to approximately 18 years of age. Deviations from linearity were modest compared with intrinsic variability (see "Discussion"). 2) Pituitary volume declined slightly throughout adulthood. 3) This approach yields natural intuitive representations similar to somatic growth charts. 4) Linear representations allow simple $z$-score calculation, suitable for trend analysis, without tables or charts. Normalization with respect to age, rather than any metric of body habitus, was performed because pituitary damage might otherwise be masked by the poor growth that resulted from subsequent endocrine insufficiency. To ensure continuity of predicted $z$ scores, we performed the following iterative approach: Linear regression was performed over the pediatric $(0-18$ years $)$ and adult ages independently. These fits produced similar but not identical values at 18 years of age. The optimal intersection point was determined by linear interpolation weighted by the mean-squared residual error of each fit; thus, the better fit had more influence in determining the intersection point. Pediatric and adult linear regressions were subsequently repeated and constrained to pass through the same value at 18 years of age. Mean-square errors of the regressions were found to be similar. To prevent a discontinuity in $z$ scores at 18 years of age, a combined mean-squared error was calculated after correcting for differences in group sizes.

\section{Results}

Subjects were 19.6 \pm 11.8 years old (range, 2.0-48.1 years). Ethnicity was generally representative of Los Angeles: $49 \%$ (49/100) Hispanic, 25\% (25/100) white, 9\% (9/100) AfricanAmerican, 7\% (7/100) Armenian, and 10\% (10/100) other races (mostly Far and Middle Eastern). Sex distribution and physical characteristics divided into age groups are summarized in Table 1. There was good sex balance across all ages.

Figure 1 represents a multiecho spin-echo image for the R2 calculation at a TE of $30 \mathrm{~ms}$ (left panel). The middle panel indicates mild even-odd echo oscillation, but the T2 calculated by all echoes ( $89.9 \mathrm{~ms})$ was nearly identical to the $\mathrm{T} 2$ calculated by even echoes only $(88.6 \mathrm{~ms})$. The right-hand panel demonstrates the R2 histogram from signal-intensity decays fitted on a pixelwise basis. The median R2 was $11.3 \mathrm{~Hz}$, corresponding to a T2 decay of $88.9 \mathrm{~ms}$. All 3 approaches exhibited similar $\mathrm{T} 2$ agreement among the subjects. However, nomograms were calculated by using pixelwise fits because iron deposition is heterogeneous in patients with iron overload, and pixelwise techniques are more appropriate in such situations. ${ }^{20}$ We also chose to fit the full 8-echo decay, rather than using only even echoes, because finer echo timing is necessary to accurately 


\begin{tabular}{|c|c|c|c|c|c|}
\hline $\begin{array}{l}\text { Age Range } \\
\text { (yr) }\end{array}$ & Sex & Height $(\mathrm{cm})$ & Weight $(\mathrm{kg})$ & BSA $\left(m^{2}\right)$ & BMI $\left(\mathrm{kg} / \mathrm{m}^{2}\right)$ \\
\hline$\overline{0-10}$ & $14 \mathrm{~F} ; 12 \mathrm{M}$ & $125.3 \pm 20.1$ & $23.6 \pm 8.6$ & $0.9 \pm 0.2$ & $15.7 \pm 3.2$ \\
\hline 10-20 & $16 \mathrm{~F} ; 17 \mathrm{M}$ & $164.0 \pm 12.6$ & $63.4 \pm 23.4$ & $1.7 \pm 0.4$ & $23.2 \pm 7.0$ \\
\hline $20-30$ & $11 \mathrm{~F} ; 12 \mathrm{M}$ & $171.8 \pm 11.1$ & $70.4 \pm 16.7$ & $1.8 \pm 0.3$ & $23.5 \pm 3.2$ \\
\hline 30-40 & $5 \mathrm{~F} ; 5 \mathrm{M}$ & $170.1 \pm 12.9$ & $82.5 \pm 16.0$ & $2.0 \pm 0.2$ & $28.5 \pm 4.9$ \\
\hline $40-50$ & $5 F ; 3 \mathrm{M}$ & $169.8 \pm 14.2$ & $78.7 \pm 15.9$ & $1.9 \pm 0.3$ & $27.2 \pm 3.6$ \\
\hline
\end{tabular}

Note:-BSA indicates body surface area; BMI, body mass index.
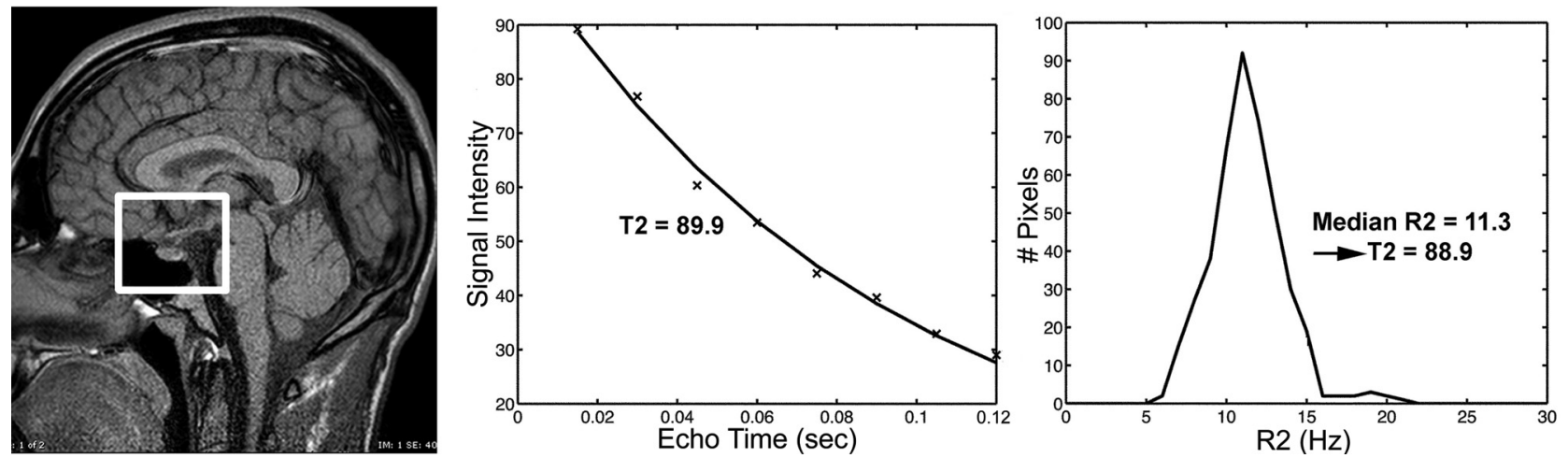

Fig 1. Pituitary R2 calculation. Left: Sagittal spin-echo image obtained at TE $15 \mathrm{~ms}$. The pituitary gland is highlighted by the white box in the first image. Middle: Signal-intensity-decay curve calculated from a region of interest including the entire anterior pituitary. Solid line represents a fit to a monoexponential without constant offset correction. Right: R2 histogram of R2 values derived for every pixel in the anterior pituitary region of interest. Median pixelwise R2 corresponds to a T2 value of 88.9 , which is within $1.1 \%$ of values calculated from a nonpixelwise technique.
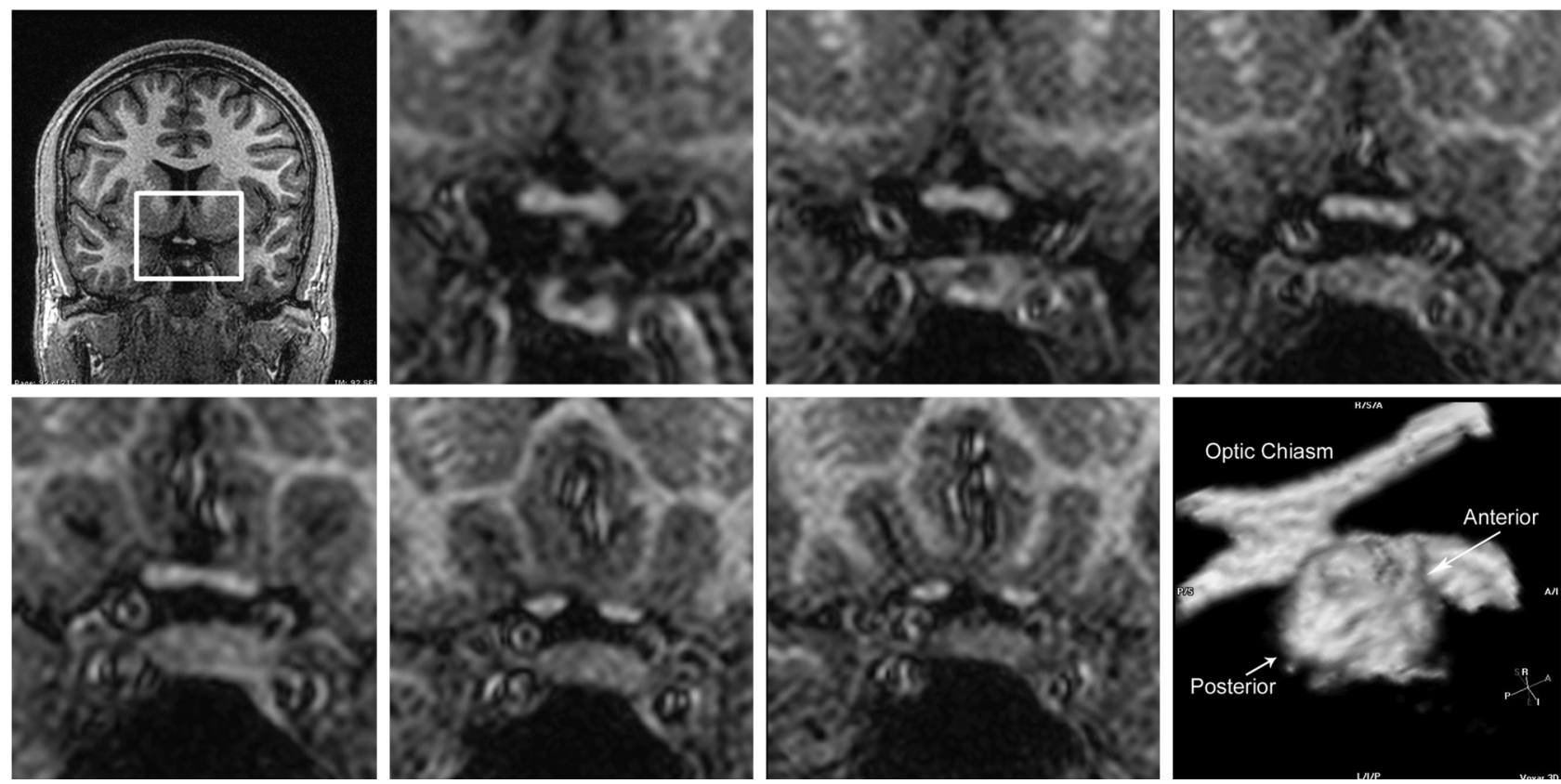

Fig 2. Pituitary volume imaging. Coronal images from the volumetric spoiled gradient-echo image acquisition, arranged from posterior to anterior (left to right and top to bottom). Every second section is shown. The bottom-right panel represents a posteroinferior-right lateral view of a 3D rendering of the pituitary gland by using level-based thresholding and limited manual excision to separate the pituitary gland from adjacent structures; the optic chiasm is shown for reference.

estimate R2 in patients with iron overload having rapid signalintensity loss.

Figure 2 shows coronal gradient-echo images for the same volunteer; the pituitary gland spans 13 sections, so every other section is displayed. The posterior pituitary gland is observed in the first 3 images across the top. It is distinguishable by its brighter signal intensity and round shape. The bottom-right image is a $3 \mathrm{D}$ reconstruction of the pituitary gland, demon- strating that the posterior pituitary gland joins the anterior pituitary gland posteroinferiorly. Some coronal images contain contributions from both regions. Demarcation was clear in approximately $90 \%$ of individuals, but separation of the anterior/posterior pituitary gland was subjective in some cases.

Median pituitary R2 rose weakly with age $\left(r^{2}=0.19\right.$, $P<.0001$ ) (Fig 3). R2 values from 6 subjects were outside the 


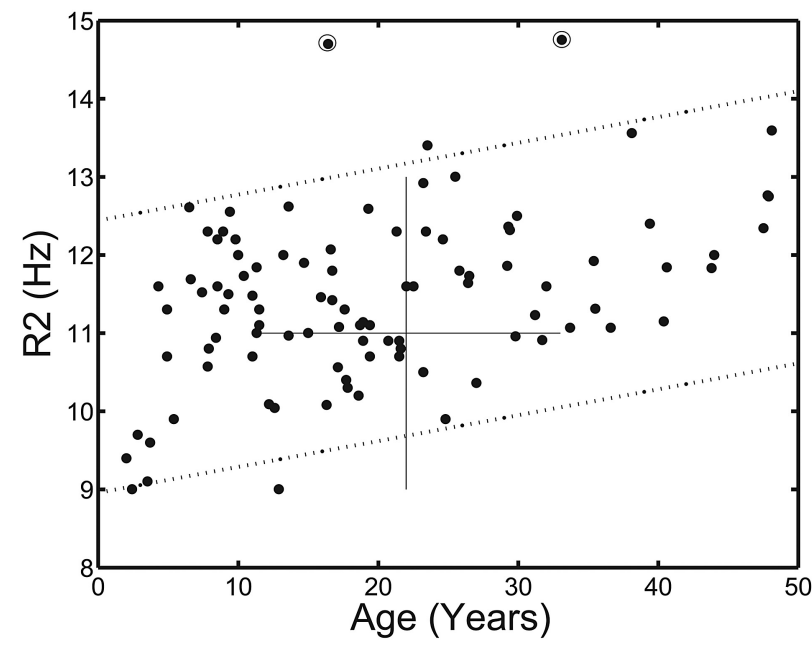

Fig 3. Sagittal R2 as a function of age. R2 increases roughly $1 \mathrm{~Hz}$ every 30 years and is sex-independent. Outlier with R2 of 17.6 (not shown) and 2 other outliers (circled) are excluded from the linear regression; the remaining subjects are normally distributed. Historical norms ${ }^{7}$ are indicated by the crossed solid lines.

95\% confidence intervals (5 were predicted). Three of these were extreme outliers (including 1 outside the graph) for no obvious reason, but the rest of the $\mathrm{R} 2$ values were normally distributed. Solid lines indicate historical 95\% confidence intervals estimated from Argyropoulou et al, ${ }^{7}$ demonstrating excellent intercenter agreement. With the mean and SD provided in Table 2, sagittal R2 $z$ scores were $0.04 \pm 1.0$, representing statistically insignificant bias. No sex differences were present. Mean and median R2 values were essentially identical (zero bias; coefficient of variation, 2.4\%). Pituitary R2 values collected in a coronal plane through the anterior pituitary gland were significantly higher than those collected in a sagittal plane $(0.4 \pm 0.8 \mathrm{~Hz}$ larger, $P<.0001)$, independent of age or sex.

Anterior and total pituitary volumes were higher in females than in males, thus sex-specific nomograms were calculated. Figure 4 demonstrates anterior pituitary volume as a function of age for both males and females (left and right). Data were well-described by a piecewise continuous linear model. Regression equations and residual error for calculated $z$ scores are summarized in Table 2 . A total of 8 subjects had measured volumes outside the $95 \%$ confidence intervals (3 more than expected), but the remaining values were normally distributed. Excluding the 2 largest outliers, the calculated $z$ score was $-0.17 \pm 1.1$, which was unbiased and independent of age and sex. Anterior pituitary volumes in females were $71 \mathrm{~mm}^{3}$ higher than those in males $\left(525 \pm 183 \mathrm{~mm}^{3}\right.$ versus $\left.454 \pm 168 \mathrm{~mm}^{3}, P=.05\right)$. Because our sampling was somewhat sparse in children younger than 6 years of age, it is not possible to extrapolate the fit accurately to birth. Solid lines depict 95\% confidence intervals derived from $>100$ children younger than 10 years of age by Fink et al. ${ }^{15}$ Our data from the present study are consistent with these historical norms, but our simple linear model overestimates pituitary volume in children younger than 10 years of age, and the confidence intervals are larger because of the inadequate sampling at the younger ages. ${ }^{15}$

Because the anterior pituitary gland is the dominant contributor to total pituitary volume, total pituitary volume has nearly identical characteristics (not shown). Excellent intercenter agreement was observed with the results derived by Takano et al in 199 pediatric subjects. ${ }^{16}$ Total pituitary measurements have slightly narrower confidence intervals, with a coefficient of variation of $13.9 \%$ at 18 years of age, compared with $16.2 \%$ for anterior pituitary volume measurements. Total pituitary volume has better reproducibility because it is not necessary to manually segment the anterior and posterior pituitary gland boundaries.

The posterior pituitary gland was the most sexually dimorphic in its size and growth pattern (not shown). Males exhibited larger posterior pituitary volumes $\left(103 \pm 41.8 \mathrm{~mm}^{3}\right.$ versus $\left.86.1 \pm 26.3 \mathrm{~mm}^{3}, P=.02\right)$. This difference was not present in young children but peaked during adolescence and early 20s, remitting slowly thereafter. Variability was quite high, however, particularly for adult males. Because iron is not deposited in the posterior pituitary, we did not formulate R2 nomograms for this region.

The potential clinical importance of establishing normative data for R2 and volume is demonstrated in Fig 5. We show 2 cases of patients with thalassemia major, matched by age and sex, who had 3 pituitary MR images during the course of 2 years; data are plotted on nomograms derived from Table 2. One patient (marked by X) was incompletely compliant with chelation therapy, leading to marked increases in her pituitary $\mathrm{R} 2$. The patient initially had high-normal anterior pituitary volume, but gland volume decreased within the normal range during 2 years. The other patient (marked by an open circle) was more compliant with chelation. Her pituitary R2 was initially increased but normalized during a 2 -year period. Anterior pituitary volume also trended favorably within the normal range. While both patients have similar normal anterior pituitary volumes at the most recent MR imaging visit, their trends suggest a markedly different prognosis.

Regression results for average height and biplane volume estimates are also shown in Table 2 and provide simpler measurements for estimating pituitary volume; 5/6 area-length volume estimates were highly correlated with both anterior and total pituitary volumes $\left(r^{2}\right.$ was 0.73 and 0.70 , respectively, $P<$.0001). Sex differences were not observed, but anterior pituitary biplane estimates underestimated planimetered values by $7.4 \pm 2.1 \%(P<.0007$ by Bland-Altman analysis $)$; total pituitary biplane estimates were unbiased with respect to planimetered values. Average pituitary height was also correlated with planimetered anterior and total pituitary volume $\left(r^{2}\right.$ was 0.53 and 0.49 , respectively, $P<.0001$ ). The coefficients of variation for average pituitary height, anterior volume (biplane), and total volume (biplane) at age 18 were $11.3 \%$, $16.0 \%$, and $14.9 \%$, respectively, comparable with the volumetric variability by planimetry.

\section{Discussion}

The purpose of this study was to characterize pituitary volume and iron (R2) by using MR imaging in a population of healthy controls, particularly in peripubertal subjects, to recognize preclinical R2 overload and volume loss in chronically transfused patients. ${ }^{13}$ Increased pituitary R2 and gland destruction are common in chronically transfused patients, causing $\mathrm{HH}$ in approximately $50 \%$ of patients with thalassemia major. ${ }^{2,4,5}$ The clinical effects of gland damage cannot be seen until after 


\begin{tabular}{|c|c|c|c|c|}
\hline & \multicolumn{2}{|c|}{ Pediatric } & \multicolumn{2}{|c|}{ Adult } \\
\hline & Mean & SD & Mean & SD \\
\hline \multicolumn{5}{|l|}{ Males } \\
\hline Anterior volume $\left(\mathrm{mm}^{3}\right)$ & $27.5 A+69.7$ & 94.4 & 586.1-1.19 A & 94.4 \\
\hline Posterior volume $\left(\mathrm{mm}^{3}\right)$ & $5.66 A+30.9$ & $21.8 \%$ & $152.6-1.1 \mathrm{~A}$ & 37 \\
\hline Total volume $\left(\mathrm{mm}^{3}\right)$ & $33.6 A+95.8$ & 98 & $756.6-2.81 \mathrm{~A}$ & 98 \\
\hline Sagittal R2 $(\mathrm{Hz})$ & $0.0331 A+10.7$ & 0.88 & Same & Same \\
\hline \multicolumn{5}{|l|}{ Females } \\
\hline Anterior volume $\left(\mathrm{mm}^{3}\right)$ & $29.3 A+116.9$ & 101.5 & $659-.816 \mathrm{~A}$ & 101.5 \\
\hline Posterior volume $\left(\mathrm{mm}^{3}\right)$ & $1.59 A+60$ & 22.5 & $0.96 A+71.3$ & 22.5 \\
\hline Total volume $\left(\mathrm{mm}^{3}\right)$ & $32.2 \mathrm{~A}+171.5$ & 106.5 & $795-1.84 \mathrm{~A}$ & 106.5 \\
\hline Sagittal R2 (Hz) & $0.0331 A+10.7$ & 0.88 & Same & Same \\
\hline \multicolumn{5}{|l|}{ Volume estimates } \\
\hline Average height & $0.142 A+3.21$ & 0.65 & $6.17-0.0219 \mathrm{~A}$ & 0.65 \\
\hline 5/6 Area-length (ant.) & $28.9 A+54.7$ & 91.6 & 586.1-0.668 A & 91.6 \\
\hline 5/6 Area-length (total) & $33.8 A+150.1$ & 112.6 & $798.1-2.24$ A & 112.6 \\
\hline
\end{tabular}

Note:-A indicates age in years; ant., anterior.
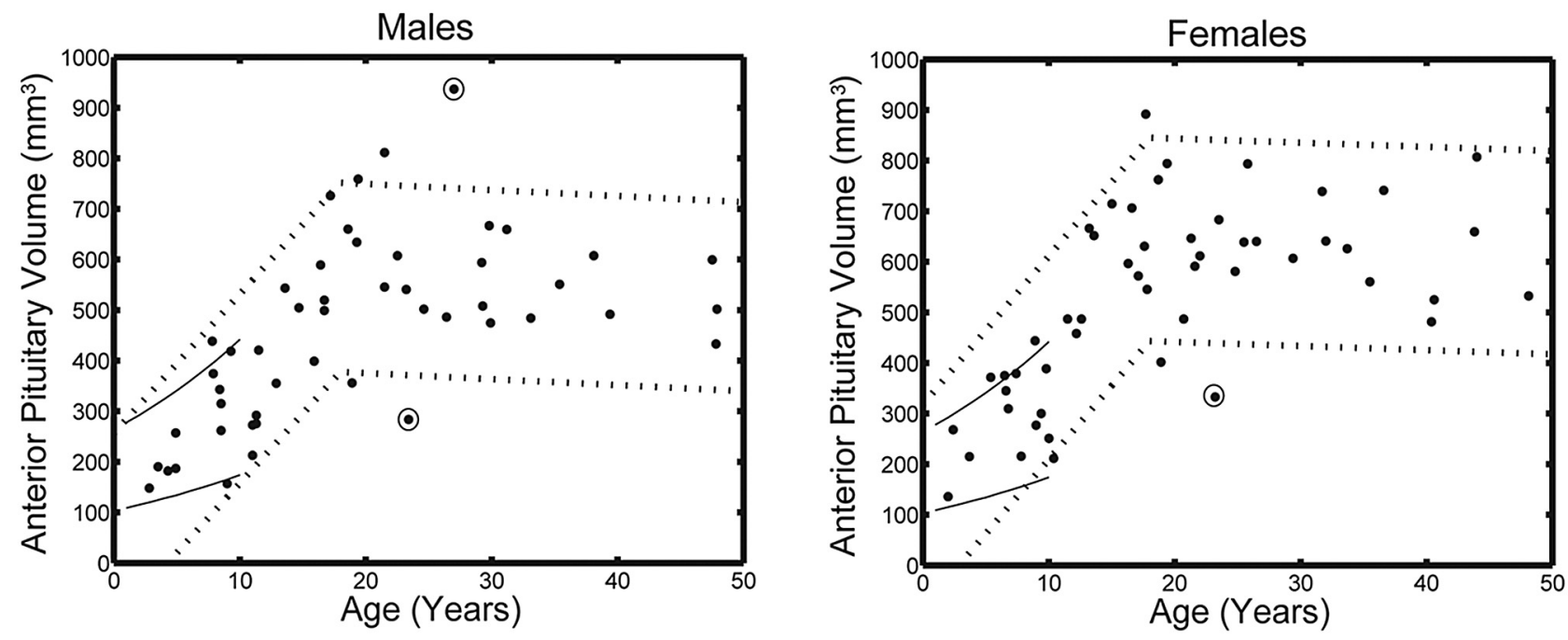

Fig 4. Anterior pituitary volume as a function of age. Males are shown on the left and females on the right. Data are well-described by piecewise linear regression formulas, with a hinge point at 18 years of age. Data are normally distributed but require outlier exclusion (circles). Historic norms for children $<10$ years of age 15 are indicated by the solid lines.

puberty and may be irreversible. Although some studies have shown long-term reversibility of clinical effects with aggressive iron-chelation therapy, ${ }^{21}$ this recovery is most likely restricted to patients who have glandular iron without concomitant apoptosis and volume loss. ${ }^{21}$ Therefore, it is advantageous to reverse R2 overload before volume loss and clinical symptoms occur. ${ }^{6,12}$ There is currently little literature reporting normative data for R2. ${ }^{7,22}$ Additionally, we aimed to supplement current pituitary volume literature ${ }^{14-16}$ by deriving sex-appropriate $z$-score calculations for pediatric and adult controls.

The upward drift of $\mathrm{R} 2$ with age ( $1 \mathrm{~Hz}$ every 30 years) may represent iron accumulation, similar to iron accumulation in brain nuclei, ${ }^{23}$ but changes in cell cellularity and water content cannot be excluded. Similarly, the broad confidence intervals $( \pm 2 \mathrm{~Hz}$ ) likely represent contributions from iron, water, and protein content. In patients with iron overload, the pituitary gland has relatively light iron deposition compared with other vulnerable organs such as the liver, heart, and pancreas. ${ }^{24}$ Nonetheless, the $95 \%$ confidence intervals observed in our study (between 12.5 and $14.1 \mathrm{~Hz}$ ) are well below those observed in patients with thalassemia major. ${ }^{7}$ Argyropoulou et $\mathrm{al}^{7}$ demonstrated that pituitary $\mathrm{R} 2$ was $17 \pm 4 \mathrm{~Hz}$ in patients with normal gonadotropin-stimulation test results, while it was $24 \pm 6 \mathrm{~Hz}$ in patients with biochemical or clinical $\mathrm{HH}$. These data indicate that pituitary R2 measurements convey a graded risk for pituitary dysfunction and that normal intersubject variability is small compared with the R2 changes associated with clinical disease.

R2 can be calculated by either fitting an exponential to average pixel values in a region (region-of-interest-based) or by averaging the $\mathrm{R} 2$ values calculated at each pixel in the region (pixelwise). We found the differences to be negligible in healthy subjects. Similarly, pixelwise distributions were relatively symmetric, as was seen by statistically identical mean and median values. However, R2 can be quite heterogeneously distributed in patients with iron overload, and a region-based method does not produce a good fit to a monoexponential function. In such patients, the mean or median R2 calculated from pixelwise fits is a more robust metric of the total iron concentration. ${ }^{20}$ Therefore, we used pixelwise fits for this normal population. R2 estimates collected in the coronal plane 

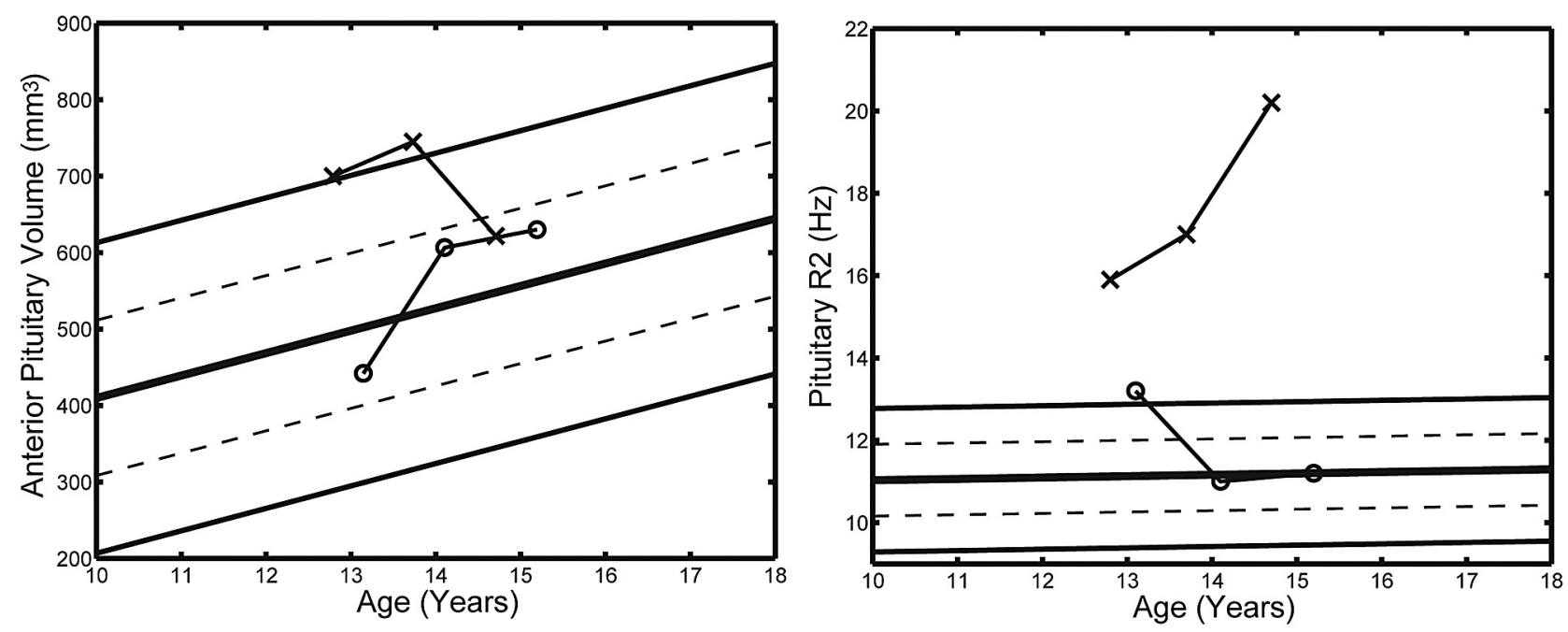

Fig 5. Clinical use of normative data. Anterior pituitary volume and pituitary R2 trends are shown for 2 patients with thalassemia major. These trends are during a 2-year period. One patient (0) was compliant with chelation and showed improvement of pituitary R2 and volume $z$ scores. The other patient (X) was less compliant, and pituitary R2 and anterior pituitary volume z scores worsened.

were slightly biased compared with the sagittal plane $(0.4 \mathrm{~Hz}$ higher). This finding reflects greater weighting of the anterior portion of the gland, but this disparity is small and systematic.

Although cardiovascular volumes are best normalized to body surface area, normalization of pituitary volume was done with respect to age because pituitary size and health directly affect patient growth. Pituitary volume increased with age up to 18 years, after which it plateaued and even slightly decreased, consistent with changes in other organs. ${ }^{25-27}$ Most interesting, females had larger anterior and total pituitary volumes than males. The etiology is unknown, but lactotrophs are the most common cell type in the anterior pituitary gland, making up approximately one-sixth of glandular volume. ${ }^{28}$ Premenopausal females have more lactotrophs (starting in infancy) and produce an average of 33\% more prolactin than males. ${ }^{28}$ Premenopausal females may also lose volume less quickly, because estrogen protects against oxidative stress in the brain. ${ }^{29}$ Regardless of the etiology, this size discrepancy is important to properly identify pituitary volume loss in patients with iron overload.

Simplified estimates of pituitary volume (by average height or biplane measurements) correlated well with planimetered anterior and total pituitary volumes and had acceptable bias and variability. No sex differences were identified, suggesting intrinsically lower sensitivity to physiologic differences than planimetered techniques. However, studies in subjects with iron overload will be necessary to determine whether these simplified metrics are sufficiently sensitive and stable to stratify the risk of pituitary dysfunction. Our regression equations in Table 2 provide easy calculation of $z$ scores, which can help assess the utility of these estimates in future studies.

The strength of this study is its broad balanced sampling with respect to age and sex and the simplified $z$-score estimation. With these estimation metrics, pituitary $\mathrm{R} 2$ and volumetric $z$ scores can be easily calculated and trended with time, similar to growth charts. The potential importance of establishing normative data is illustrated in Fig 5. We postulate that trends in R2 and volume, even more than individual measurements, will identify patients having the greatest risk for clinical problems as well as their response to intensified chelation therapy. Large prospective studies in patients at risk will be necessary to test this hypothesis.

Unfortunately, simplicity forfeits some sensitivity. The "true" anterior pituitary curve is at least triphasic, with a shallow age-mediated rise from birth to 9 or 10 years of age, a sex-specific rapid rise in gland volume through puberty, and sex-specific volume loss during adulthood. With the intersubject variability demonstrated in ours and other studies, it would take approximately 500 subjects to capture these dynamics in a single ethnic population and more in a multicultural environment such as Los Angeles. Nonetheless, our derived confidence intervals agree quite well with previously published norms ${ }^{15,16}$ and suggest that the loss in sensitivity is small, particularly given that pituitary height is reduced $>50 \%$ in symptomatic patients with iron overload. ${ }^{6}$

Another limitation of our study is the failure to ethnicitymatch our nomograms to the patients with iron-overload disorders, which is a tremendously ethnically diverse population. Our cohort of subjects represents a convenience sample from the greater Los Angeles area. This limitation may introduce bias between our $z$ scores and those of specific target populations. It may also broaden the confidence intervals because pubertal timing is influenced by ethnicity and body composition. This is analogous to the mismatch between conventional growth charts and most immigrant populations. Nonetheless, serial trends in $z$ scores should be diagnostic, particularly if a baseline examination is obtained before there is a priori concern for volume loss. Our preliminary work in patients with thalassemia major has suggested that volume loss does not manifest until the second decade of life. ${ }^{13}$

Data from 7 of our youngest patients were obtained as piggy-back examinations on children undergoing clinically indicated MR imaging for seizures or developmental delay. We could not ethically obtain data from children this age by any other means. Although these children had normal brain morphology, we cannot be certain that there is not selection bias between the referral diagnosis and pituitary size. Nonetheless, 
our data were internally consistent with data from the other subjects and with previously published data.

The specificity of R2 elevations and pituitary loss is also imperfect. While data were predominantly normally distributed, both measurements had a few notable outliers. The etiology of the 3 increased R2 values (of 100) was unknown. Outliers in the anterior and posterior volume estimates were best explained by inadequate manual segmentation because total pituitary volume estimates were significantly more robust. Nonetheless, 2 subjects had markedly decreased total pituitary volumes. In these subjects, there was superior intrusion of an arachnoid cyst through a defect in the roof of the sella turcica (forme fruste of primary empty sella syndrome). ${ }^{30} \mathrm{De}-$ spite these limitations, these data represent a critical step toward detection of preclinical R2 deposition and volume loss in transfusional siderosis.

\section{Conclusions}

We derived sex- and age-specific $z$ scores for pituitary R2, anterior pituitary volume, and total pituitary volume. Values derived from these equations are continuous with respect to age, making them suitable for serial evaluation. Our observed norms were consistent with previously published pediatric data, suggesting clinically adequate intercenter reproducibility. Intersubject variability in R2 and volume estimates is smaller than the changes in pituitary $\mathrm{R} 2$ and size observed in subjects with iron overload with endocrine dysfunction. Thus, we postulate that our normative equations will be useful for detection of preclinical iron deposition and volume loss in chronically transfused patients.

\section{Acknowledgments}

We are indebted to Arthur Kohatsu for his assistance in recruiting and scanning healthy subjects.

Disclosures: Thomas D. Coates-Research Support (including provision of equipment or materials): Novartis, Details: support for research related to sickle cell and iron overload; salary and supplies for clinical study; terminated in 2010; Speaker Bureau: Novartis, Details: Spoke concerning the use of Exjade for iron overload in Sickle Cell disease and thalassemia. SCD in general; $\$ .00$ in $2010 \ldots \$ .00$ in 2011 to date; Consultant. Novartis, Details: consulted concerning iron overload; $\$ 2000$ in 2011. John Wood-Research Support (including provision of equipment or materials): Novartis, Details: 1) investigator-initiated research grant titled "Evaluation of the effects of vitamin $\mathrm{C}$ and deferasirox in iron loaded animals." Role: principal investigator; 2) investigator-initiated research grant, "Changes in pituitary iron and volume with deferasirox therapy in transfusional iron overload." Role: principal investigator; Consultant. Ferrokin Biosciences, Details: core lab director and medical consultant to a Phase II trial of FBS0701 in subjects with iron overload; ApoPharma, Details: MRI and medical consultant.Other Financial Relationships: Novartis, Details: travel support for advisory panels; no direct remuneration.

\section{References}

1. Hershko C, Link G, Cabantchik I. Pathophysiology of iron overload. Ann N Y Acad Sci 1998;850:191-201

2. Borgna-Pignatti C, Rugolotto S, De Stefano P, et al. Survival and complications in patients with thalassemia major treated with transfusion and deferoxamine. Haematologica 2004;89:1187-93

3. Angelucci E, Brittenham GM, McLaren CE, et al. Hepatic iron concentration and total body iron stores in thalassemia major. N Engl J Med 2000;343:327-31
4. Vogiatzi MG, Macklin EA, Trachtenberg FL, et al. Differences in the prevalence of growth, endocrine and vitamin $\mathrm{D}$ abnormalities among the various thalassanemia syndromes in north America. Br J Haematol 2009;146:546-56

5. De Sanctis V. Growth and puberty and its management in thalassaemia. Horm Res 2002;58(suppl 1):72-79

6. Argyropoulou MI, Kiortsis DN, Metafratzi Z, et al. Pituitary gland height evaluated by MR in patients with beta-thalassemia major: a marker of pituitary gland function. Neuroradiology 2001;43:1056-58

7. Argyropoulou MI, Metafratzi Z, Kiortsis DN, et al. T2 relaxation rate as an index of pituitary iron overload in patients with beta-thalassemia major. $A J R$ Am J Roentgenol 2000;175:1567-69

8. Wood JC, Enriquez C, Ghugre N, et al. MRI R2 and R2* mapping accurately estimates hepatic iron concentration in transfusion-dependent thalassemia and sickle cell disease patients. Blood 2005;106:1460-65

9. Noetzli LJ, Papudesi J, Coates TD, et al. Pancreatic iron loading predicts cardiac iron loading in thalassemia major. Blood 2009;114:4021-26

10. Christoforidis A, Haritandi A, Perifanis V, et al. MRI for the determination of pituitary iron overload in children and young adults with beta-thalassanemia major. Eur J Radiol 2007;62:138-42. Epub 2006 Dec 11

11. Berkovitch M, Bistritzer T, Milone SD, et al. Iron deposition in the anterior pituitary in homozygous beta-thalassemia: MRI evaluation and correlation with gonadal function. J Pediatr Endocrinol Metab 2000;13:179-84

12. Hekmatnia A, Radmard AR, Rahmani AA, et al. Magnetic resonance imaging signal reduction may precede volume loss in the pituitary gland of transfusion-dependent beta-thalassemic patients. Acta Radiol 2010;51:71-77

13. Wood JC, Noetzl L, Hyderi A, et al. Predicting pituitary iron and endocrine dysfunction. Ann N Y Acad Sci 2010;1202:123-28

14. Marziali S, Gaudiello F, Bozzao A, et al. Evaluation of anterior pituitary gland volume in childhood using three-dimensional MRI. Pediatr Radiol 2004;34: 547-51

15. Fink AM, Vidmar S, Kumbla S, et al. Age-related pituitary volumes in prepubertal children with normal endocrine function: volumetric magnetic resonance data. J Clin Endocrinol Metab 2005;90:3274-78

16. Takano K, Utsunomiya $\mathrm{H}$, Ono $\mathrm{H}$, et al. Normal development of the pituitary gland: assessment with three-dimensional MR volumetry. AJNR Am J Neuroradiol 1999;20:312-15

17. Safarinejad MR. Evaluation of semen quality, endocrine profile and hypothalamus-pituitary-testis axis in male patients with homozygous betathalassemia major. J Urol 2008;179:2327-32

18. Safarinejad MR. Reproductive hormones and hypothalamic-pituitaryovarian axis in female patients with homozygous beta-thalassemia major. J Pediatr Hematol Oncol 2010;32:259-66

19. Karabulut A, Balci Y, Demirlenk S, et al. Gonadal dysfunction and pelvic sonographic findings in females with thalassaemia major. Gynecol Endocrinol 2010;26:307-10

20. Ghugre NR, Enriquez CM, Coates TD, et al. Improved R2* measurements in myocardial iron overload. J Magn Reson Imaging 2006;23:9-16

21. Farmaki K, Tzoumari I, Pappa C, et al. Normalisation of total body iron load with very intensive combined chelation reverses cardiac and endocrine complications of thalassaemia major. Br J Haematol 2010;148:466-75

22. Argyropoulou MI, Kiortsis DN, Astrakas L, et al. Liver, bone marrow, pancreas and pituitary gland iron overload in young and adult thalassemic patients: a T2 relaxometry study. Eur Radiol 2007;17:3025-30

23. Rodrigue KM, Haacke EM, Raz N. Differential effects of age and history of hypertension on regional brain volumes and iron. Neuroimage 2011;54:750 59. Epub 2010 Oct 20

24. Lam WW, Au WY, Chu WC, et al. One-stop measurement of iron deposition in the anterior pituitary, liver, and heart in thalassemia patients. J Magn Reson Imaging 2008;28:29-33

25. Liu RS, Lemieux L, Bell GS, et al. A longitudinal study of brain morphometrics using quantitative magnetic resonance imaging and difference image analysis. Neuroimage 2003;20:22-33

26. Scahill RI, Frost C, Jenkins R, et al. A longitudinal study of brain volume changes in normal aging using serial registered magnetic resonance imaging. Arch Neurol 2003;60:989-94

27. Saisho Y, Butler AE, Meier JJ, et al. Pancreas volumes in humans from birth to age one hundred taking into account sex, obesity, and presence of type- 2 diabetes. Clin Anat 2007;20:933-42

28. Malmed S. The Pituitary. Malden, Massachusetts: Blackwell Science; 2002

29. Behl C, Skutella T, Lezoualc'h F, et al. Neuroprotection against oxidative stress by estrogens: structure-activity relationship. Mol Pharmacol 1997:51:535-41 30. Naing S, Frohman LA. The empty sella. Pediatr Endocrinol Rev 2007;4:335-42 